\title{
INFESTAÇÃO POR ANCILOSTOMÍDEOS E TOXOCARÍDEOS EM CÃES E GATOS APREENDIDOS EM VIAS PÚBLICAS, SÃO PAULO (BRASIL)
}

\author{
Valdson de Angelis Côrtes* \\ Gil Vianna Paim** \\ Rufino Antunes de Alencar Filho***
}

\begin{abstract}
CÔRTES, V. de A. et al. Infestação por ancilostomídeos e toxocarídeos em cães e gatos apreendidos em vias públicas, São Paulo (Brasil) Rev. Saúde públ., S. Paulo, 22:341-3, 1988.

RESUMO: Foram examinadas fezes, coletadas após o sacrifício, de 9.150 cães e 674 gatos, capturados nas vias públicas do Município de São Paulo, SP (Brasil), visando o encontro de ovos e parasitos das famílias Ancylostomidae e Ascaridae. A coleta do material ocorreu durante os anos de 1980-1985. As seguintes taxas de infestação foram observadas: $59,83 \%$ dos cães e $22,26 \%$ dos gatos estiveram positivos para ovos de Ancylostoma sp., enquanto que $11,70 \%$ dos cães e $17,65 \%$ dos gatos apresentaram ovos de Toxocara sp.
\end{abstract}

UNITERMOS: Doenças do cão, parasitologia. Doenças do gato, parasitologia. Fezes, parasitologia. Ancilostomíase, veterinária. Toxocaríase, veterinária.

\section{INTRODUÇÃO}

A ancilostomíase e a toxocaríase de animais de estimação (helmintoses intestinais de cães e gatos) vêm sendo reconhecidas ultimamente, e cada vez mais, como causa principal de um importante problema de saúde pública: a larva migrans cutânea e a larva migrans visceral.

$\mathrm{Na}$ literatura médica brasileira é freqüente deparar-se com artigos relatando surtos humanos de larva migrans ou abordando outros aspectos de casos humanos como características clínicas e laboratoriais ou a efetividade de drogas usadas no tratamento $1,4,5,6,8$.

Devido ao reconhecimento da importância em saúde pública das larvas migrans, vem se alertando, principalmente nos países desenvolvidos, sobre a necessidade de controle da poluição de lugares públicos (praias, "campings", tanques de areia, praças) por fezes de cães e ga$\operatorname{tos}^{2}, 9$.

A finalidade do presente artigo é comunicar alguns achados sobre infestação de Ancylostoma sp. e de Toxocara sp. em cães e gatos do Município de São Paulo.

\section{MATERIAL E MÉTODOS}

Após o sacrifício de cães e gatos que haviam sido capturados nas vias públicas do Município de São Paulo, procedeu-se à coleta de fezes desses animais, as quais foram consecutivamente examinadas pelo método direto, pelo método de flutuação em solução saturada de cloreto de sódio (método de Willis) e pelo método de sedimentação, objetivando a pesquisa de ovos de parasitos das famílias: Ancylostomidae e Ascaridae.

A coleta do material ocorreu durante o período de 1980 a 1985 , tendo sido examinadas fezes de 9.150 cães e 940 gatos.

\section{RESULTADOS E DISCUSSĀO}

$\mathrm{Na}$ Tabela 1 observa-se que do total de cães examinados, $5.420 \quad(59,83 \%)$ apresentaram ovos de ancilostomídeos e $1.071(11,70 \%)$ de toxocarídeos. A maior freqüência de positividade para ovos de ancilostomídeos foi observada no ano de 1983 com $69,62 \%$, enquanto que pa$\mathrm{ra}$ os ovos de toxocarídeos a maior frequiência foi a de 1980 com $17,29 \%$.

$\mathrm{Na}$ Tabela 2 vê-se que 150 gatos $(22,26 \%)$ estavam positivos para ovos de Ancylostoma sp. e $119(17,65 \%)$ para ovos de Toxocara sp. A maior frequiência de positividade para ovos de

\footnotetext{
* Departamento de Higiene Veterinária e Saúde Pública da Faculdade de Medicina Veterinária e Zootecnia da UNESP Botucatu - 18610 - São Paulo, SP, Brasil.

** Departamento de Prática de Saúde Pública da Faculdade de Saúde Pública da Universidade de São Paulo - Avenida Dr. Arnaldo, 715 - 01255 - São Paulo, SP - Brasil.

*** Centro de Controle de Zoonoses da Secretaria de Higiene e Saúde da Prefeitura Municipal de São Paulo - Rua Santa Eulália, 86 - 02031 - São Paulo, SP - Brasil.
} 
ancilostomídeos deu-se em 1981 com 29,07\%, enquanto que para ovos de toxocarídeos deu-se em 1984 com $21,05 \%$.

Procurando cotejar estes resultados com os de outros autores brasileiros, encontramos que a freqüência de infestação por nós observada foi, de um modo geral, mais baixa que a relata. da para outras localidades.
Zago Filho e Barreto ${ }^{12}$ (1957) através de necrópsias realizadas em 81 cães e 196 gatos de Ribeirão Preto, SP, relataram as taxas de infestação seguintes: $A$. caninum $98,76 \%, A$. braziliense $82,71 \%$ e $T$. canis $41,9 \%$, estes os resultados nos cães; e $A$. caninum $36,73 \%, A$. brasiliense $70,91 \%$ e $T$. cati $48,97 \%$, os resultados nos gatos. Carneiro e col. ${ }^{3}$ (1973) relataram o

TABELA 1

Encontro de ovos de Ancilostoma sp. e Toxocara sp. em cães apreendidos em vias públicas da cidade de São Paulo, 1980-1985

\begin{tabular}{lcccrr}
\hline & & \multicolumn{2}{c}{ Ancylostoma sp. } & \multicolumn{2}{c}{ Toxocara sp. } \\
Anos & Examinados & Positivos & $\%$ & Positivos & $\%$ \\
\hline 1980 & 376 & 166 & 44,15 & 65 & 17,29 \\
1981 & 824 & 418 & 50,73 & 103 & 12,50 \\
1982 & 1.178 & 677 & 57,47 & 200 & 16,97 \\
1983 & 2.693 & 1.875 & 69,62 & 283 & 10,50 \\
1984 & 2.338 & 1.418 & 60,65 & 175 & 10,48 \\
1985 & 1.741 & 866 & 49,74 & 1.071 & 10,05 \\
\hline Total & 9.150 & 5.420 & 59,83 & & 11,70 \\
\hline
\end{tabular}

TABELA 2

Encontro de ovos de Ancylostoma sp. e Toxocara sp. em gatos apreendidos em vias públicas da cidade de São Paulo, 1981-1985

\begin{tabular}{lccccc}
\hline & & \multicolumn{2}{c}{ Ancylostoma sp. } & \multicolumn{2}{c}{ Toxocara sp. } \\
Anos & Examinados & Positivos & $\%$ & Positivos & $\%$ \\
\hline 1981 & 86 & 25 & 29,07 & 12 & 13,95 \\
1982 & 99 & 26 & 26,26 & 13 & 13,13 \\
1983 & 115 & 26 & 22,60 & 21 & 18,26 \\
1984 & 133 & 34 & 25,56 & 28 & 21,05 \\
1985 & 241 & 39 & 16,18 & 45 & 18,67 \\
\hline Total & 674 & 150 & 22,26 & 119 & 17,65 \\
\hline
\end{tabular}

encontro de helmintos em Canis familiaris no Município de Goiânia, Estado de Goiás. Seus resultados derivam de 40 necrópsias e a taxa de infestação foi a seguinte: $A$. caninum $92,5 \%$, A. braziliense $67,5 \%$ e $T$. canis $17,5 \%$. Lara e col. ${ }^{7}(1981)$, através de necrópsias realizadas em 118 cães de Pelotas, Rio Grande do Sul, encontraram $60,47 \%$ dos animais com $A$. caninum, $0,41 \%$ com $A$. braziliense e $5,32 \%$ com $T$. canis.

Ressalte-se que enquanto nossos resultados foram obtidos através do exame de uma amostra de fezes, os dos trabalhos citados anteriormente foram conseguidos pelo encontro dos vermes adultos após a necrópsia. Compulsando a literatura brasileira mais recente, encontra-se o artigo de Ogassawara e col.10 (1986) que relatam os resultados de exame copro-parasitológicos em 215 gatos procedentes de diferentes áreas da cidade de São Paulo. A taxa de infestação encontrada por esses autores foi de $19,5 \%$ para Ancylostomidae e de $22,3 \%$ para Ascaridae, bastante próxima dos resultados encontrados no presente trabalho. Em outro artigo, Ogassawara e col." (1986), já agora baseados em necrópsias efetuadas em 54 gatos apreendidos em ruas da cidade de São Paulo, citam taxas de infestação mais elevadas: Toxocara cati, $53,77 \% ; T$. canis, 3,7\%; Ancylostoma sp. $25,9 \%$ e $A$. braziliense, $37,0 \%$.

\section{AGRADECIMENTOS}

À equipe do Centro de Controle de Zoonoses da Secretaria de Higiene e Saúde da Prefeitura Municipal de São Paulo pelas facilidades proporcionadas para a realização do presente trabalho. 
CÔRTES, V. de A. et al. [Ancylostoma and toxocara infestation in dogs and cats impounded on throughfares of S. Paulo, Brazil]. Rev. Saúde públ., S. Paulo, 22:341-3, 1988.

ABSTRACT: Feces, collected after the killing of 9,150 dogs and 674 cats caught on throughfares of S. Paulo City, Brazil, and impounded, were examined for evidence of Ancylostomidae and Ascaridae parasites by the direct Willis' and sedimentation methods. The present article relates to material collected during the period 1980-1985. The following infestation rates were discovered: $59.83 \%$ of dogs and $22.26 \%$ of cats were found to have Toxocara sp. ova.

UNITERMS: Dog diseases, parasitology. Cat diseases, parasitology. Feces, parasitology. Ancylostomiasis, veterinary. Toxocariasis, veterinary.

\section{REFERÊNCIAS BIBLIOGRÁFICAS}

1. ABE-JACOB, C.M. et al. Larva migrans visceral por Toxocara canis. Rev. Ass. med. bras., 30:187-91, 1984.

2. BORG, O.A.\& WOODRUFF, A.W. Prevalence of infective ova of Toxocara species in public places. Brit. med. J., 4:470-2, 1973.

3. CARNEIRO, J.R. et al. Prevalência de helmintos em Canis familiaris no município de Goiânia Rev. Pat. trop., 2:401-4, 1973.

4. CHIATTONE, C.S. et al. Sindrome de larva migrans visceral em adulto: apresentação de um caso. Rev. Inst. Adolfo Lutz, 43:85-8, 1983.

5. GUSMÃO, Q.M.W.B. \& CARDOSO, A.E.C. Larva migrans cutis, tratamento com cambendazol. $A n$. bras. Derm., 59:226-8, 1984.

6. KANAKAMI, E. et al. Larva migrans visceral na infância: relato de dois casos. Arq. Gastroent., 21:837,1984

7. LARA, S.I:M. et al. Helmintos parasitos de canis familiaris de Pelotas-Rio Grande do Sul. Arq. Esc. Vet. UFMG, 33:293-97, 1981.
8. LIMA, W.S. et al. Surto de larva migrans cutânea em uma creche de Belo Horizonte, Minas Gerais (Brasil) Rev. Inst. Med. trop. S. Paulo, 26:122-4, 1984.

9. NYC enacts law to clean up New York streets and parks. Vet. publ. Hith Notes, (Jul.):5, 1978.

10. OGASSAWARA, S. et al. Prevalência de endoparasitos em gatos na cidade de São Paulo. Rev. Fac. Med. vet. Zootec. Univ. S. Paulo, 23:31-8, 1986.

11. OGASSAWARA, S. et al. Prevalência de infecções helmínticas em gatos na cidade de São Paulo. Rev. Fac. Med. vet. Zootec. Univ. S. Paulo, 23:145-9, 1986.

12. ZAGO FILHO, H. \& BARRETO, M.P. Estudo sobre a prevalência e intensidade de infestação por helmintos intestinais em cães e gatos de Ribeirão Preto, SP. Rev. bras. Malar., 9:295-304, 1957.

Recebido para publicação em 26/10/1987 Reapresentado em 29/4/1988

Aprovado para publicação em 5/5/1988 